\title{
Discrete Fourier transform based frequency characteristics of iterative learning control for linear discrete-time systems
}

\author{
Xiaohui Li' and Xiaoe Ruan ${ }^{1 *}$ (D)
}

"Correspondence:

wruanxe@xjtu.edu.cn

${ }^{1}$ School of Mathematics and

Statistics, Xi'an Jiaotong University,

Xi'an, P.R. China

\section{Springer}

\begin{abstract}
For discrete-time iterative learning control systems, the discrete Fourier transform (DFT) is a powerful technique for frequency analysis, and Toeplitz matrices are a typical tool for the system input-output transmission. This paper first exploits $z$-transform and DFT-based frequency properties for iterative learning control systems and studies the convergence property of a Toeplitz matrix to the power of iteration index. The exploitation exhibits that for the finite-length discrete-time iterative learning control systems, the time-domain convolution theorem for the $z$-transform and DFT is no longer true, and the Toeplitz matrix to the power of iteration index converges if and only if the identical diagonal element lies in the unit circle. Then, by considering the DFT to a finite-length sequence as a linear transform, it is easy to equivalently reform the input-output equation of linear discrete time-invariant and time-varying ILC systems as an algebraic discrete-frequency equation. Thus the derivative-type (D-type) iterative learning control (ILC) converges in a discrete-frequency domain if and only if it converges in a discrete-time domain. Numerical simulations are carried out to exhibit the validity and effectiveness.
\end{abstract}

Keywords: Iterative learning control; Discrete Fourier transform; Monotone convergence; Linear discrete systems; Power formula

\section{Introduction}

Since the iterative learning control (ILC) has been invented three decades before, it has been acknowledged as an efficacious intelligent strategy for a robot manipulator to repetitively execute a desired trajectory tracking over a finite time interval [1-3]. The mechanism is iterative generating an upgrading control input for the next iteration by means of compensating the control input at the current iteration with its proportional, integral, and/or derivative tracking discrepancy between the current output and the desired trajectory [4-12]. The pursuing aim is that the generated control input may drive the system to track the desired trajectory as precise as possible as the iteration index goes on, or in other words, the ILC is convergent.

Reviewing the contributions of the ILC convergence for discrete-time systems, the analytical techniques are mainly the time- and frequency-domains. In terms of convergence in a discrete-time domain, the kernel idea is to express the ILC dynamics as an algebraic input-output equation by the lift vector technique, and thus the ILC convergence is equiv-

(c) The Author(s) 2019. This article is distributed under the terms of the Creative Commons Attribution 4.0 International License (http://creativecommons.org/licenses/by/4.0/), which permits unrestricted use, distribution, and reproduction in any medium, provided you give appropriate credit to the original author(s) and the source, provide a link to the Creative Commons license, and indicate if changes were made. 
alent to the stability of the transmit matrix as shown [13-26]. The idea is innovative, and the results are progressive. However, the convergence only involves the asymptotical tracking behavior measured in the sense of some fixed norm $[26,27]$ but does not much concerns with the transient performance evolution along the iteration axis. In fact, it is worth noticing that the input-output transmit matrix of a linear ILC-driven system is Toeplitz, and thus along the iteration axis the evolution behavior of the Toeplitz matrix to the power of iteration index must convey the learning performance evolution behaviors, including the transient overshooting, asymptotical convergence, or convergence monotonicity. Therefore the inherent significant property of the Toeplitz matrix is advantageous to ILC convergence analysis in a direct manner but not well discussed yet. This paper addresses it.

Despite the ILC convergence explorations in the time domain, the ILC convergence analysis in the frequency domain is necessary for filtering and cut-off frequency computation. For this, one existing analytical technique is the $z$-transform as adopted in [28-31], where the $z$-transform-based frequency-domain ILC convergence and robustness have been made basing on the postulation that the time-domain convolution theorem holds, which converts the convolution of two infinite-length discrete-time sequences into an algebraic multiplication of two $z$-transforms. However, as the input and output of a discretetime ILC-driven system are of finite length, the $z$-transform, which is fit to an infinitelength signal [32], may not precisely deliver the frequency information. In the authors' opinion, iis regarded as an approximate computation. This opinion has also been commented in [33].

Recall that the ILC system is repetitive. Then, the one-iteration ILC-driven system output can be regarded as a segment of a periodic sequence. It is thus feasible to make use of the discrete Fourier transform (DFT) to compute the output spectrum. For the regard, Owens' group has firstly introduced the DFT for error spectrum analysis [34], which has regarded the finite-length discrete-time output as a truncation of an infinite-length system output, where, by defaulting the time-domain convolution theorem for a finite-length ILC system, the frequency-wise input-output relation is described as an algebraic equation. For the circumstance, the Owens' DFT technique is still $z$-transform-based. The socalled frequency-wise algebraic equation looks so brief that it has inspired a number of frequency-relevant ILC convergence and robustness investigations [35-41]. But, by careful comparison, one acquired that the complexity and premise of the ILC convergence as well as the robustness in discrete-time domain [17] are quite different from that made by Owens' DFT technique [34] in frequency domain, so that it is hard to match their equivalence. This is not mathematically logic and very possibly makes confusion and puzzlement for practical applications. In authors' opinion, the confusion is incurred by the approximate computation of $z$-transform-based Owens' DFT technique. As a matter of fact, by reviewing the concept of $z$-transform, we see that it fits an infinite-length sequence whose spectrum at each frequency is relevant to the sequence terms at all sampling times [32]. This means that the spectrum computed by the $z$-transform-based Owens' DFT technique is just an approximation because the finite-length truncation of an infinite-length system has lost last part of information. The approximation precision is relevant to the truncation length and stability of the system. Generally speaking, for a given system, the longer the truncation length, the better the precision. However, for a fixed truncation length and 
a given input, the precision of an unstable system is worse than that of a stable system because the energy loss of the last part information of an unstable system is larger.

Actually, by virtue of the repetition feature of the ILC system, the iteration-wise output can be regarded as a segment of a periodic sequence whereas for a periodic sequence, the DFT is a well-known powerful technique, which expresses the sequence with the fundamental $N$ period as a summation of a fundamental sine wave plus $(N-1)$ harmonic sine waves. Its equivalent form is in exponential complex-variable functions. Then the frequency-domain spectrum can be precisely computed by DFT in a direct manner. This motivates the paper firstly to investigate DFT properties for linear discrete time-invariant (LDTI) derivative-type (D-type) ILC systems and the convergence of the Toeplitz matrix to the power of the iteration index. The followed works are the frequency-domain convergence derivation of LDTI D-type ILC systems and its generalization to linear discrete time-varying (LDTV) D-type ILC systems.

The rest of the paper is organized as follows. Section 2 exhibits properties of the $z$ transform and DFT for truncated LDTI systems together with convergence property of Toeplitz matrix to the power of iteration index. Section 3 presents frequency-domain convergence analysis of LDTI and LDTV D-type ILC systems, respectively. Numerical simulations are made in Sect. 4 , and Sect. 5 concludes the paper.

\section{2 z-Transform and DFT for truncated LDTI systems}

\section{1 $z$-Transform feature of truncated LDTI systems}

Definition $1\left(z\right.$-transform and inverse $z$-transform [32]) For a sequence $\{h(n)\}_{n=0}^{n=+\infty}=$ $\{h(0), h(1), \ldots, h(n), \ldots\}$, its $z$-transform is defined as

$$
\hat{h}(z)=Z(h(n))=\sum_{n=0}^{+\infty} h(n) z^{-n}, \quad R_{h_{-}}<|z|<R_{h^{+}}
$$

where $z$ is a complex variable, and $R_{h_{-}}<|z|<R_{h_{+}}$refers to the region of convergence (ROC).

Then the inverse $z$-transform of $\hat{h}(z)$ is defined as a contour integration:

$$
h(n)=Z^{-1}(\hat{h}(z))=\frac{1}{2 \pi j} \oint_{c} \hat{h}(z) z^{n-1} \mathrm{~d} z
$$

where $C$ represents a closed contour within the ROC: $R_{h_{-}}<|z|<R_{h_{+}}$and $j^{2}=-1$.

By derivation it is easy to testify the additivity and homogeneity for both $z$-transform and inverse $z$-transform.

Lemma 1 (Time-domain convolution theorem [32]) Given $\{w(n)\}_{n=0}^{n=+\infty} \stackrel{\text { def }}{=}\{h(n) *$ $v(n)\}_{n=0}^{n=+\infty}=\left\{w(n)=\sum_{l=0}^{n} h(n-l) v(l)\right\}_{n=0}^{n=+\infty}$, we have $\hat{w}(z)=\hat{h}(z) \hat{v}(z)=\hat{v}(z) \hat{h}(z)$, provided that the z-transform-based frequency domain input-output dynamics for a discrete linear time-invariant system takes the form

$$
\hat{y}(z)=\hat{g}(z) \hat{u}(z)
$$

where $\hat{g}(z), \hat{u}(z)$, and $\hat{y}(z)$ denote the $z$-transform transfer function, input, and output, respectively. 
By the time-domain convolution theorem the discrete time-domain dynamics of system (1) is equivalently formulated as

$$
y(n+1)=\sum_{l=0}^{n} g(n+1-l) u(l) \quad \text { for } n \in \mathbb{N}=\{0,1,2, \ldots\}
$$

where $y(n)=Z^{-1}(\hat{y}(z)), g(n)=Z^{-1}(\hat{g}(z))$, and $u(n)=Z^{-1}(\hat{u}(z))$.

Let $\mathbf{I}=\left[\boldsymbol{\varepsilon}_{1}\left|\boldsymbol{\varepsilon}_{2}\right| \cdots \mid \boldsymbol{\varepsilon}_{N}\right]$ denote the $N$ th-order unit matrix, and let $\mathbf{S}=\left[\boldsymbol{\varepsilon}_{2}\left|\boldsymbol{\varepsilon}_{3}\right| \cdots\left|\boldsymbol{\varepsilon}_{N}\right| \mathbf{0}\right]$ be a shift matrix.

Proposition $1 \mathbf{S}^{l}=\left[\boldsymbol{\varepsilon}_{l+1}\left|\boldsymbol{\varepsilon}_{l+2}\right| \cdots\left|\boldsymbol{\varepsilon}_{N}\right| \mathbf{0}|\cdots| \mathbf{0}\right]$ for $l=2,3, \ldots, N-1$ and $\mathbf{S}^{N}=\mathbf{0}$.

Define the truncation operator

$$
\left(\Phi_{\left[n_{1}, n_{2}\right]} x\right)(n)= \begin{cases}x(n), & n_{1} \leq n \leq n_{2} \\ 0, & \text { otherwise }\end{cases}
$$

Here the integer $\left(n_{2}-n_{1}+1\right)$ is the assigned truncation length.

Denote the lifted vectors as

$$
\begin{aligned}
& \Phi_{[1, N]} y=\left[\left(\Phi_{[1, N]} y\right)(1)\left|\left(\Phi_{[1, N]} y\right)(2)\right| \cdots \mid\left(\Phi_{[1, N]} y\right)(N)\right]^{\mathrm{T}}, \\
& \Phi_{[0, N-1]} u=\left[\left(\Phi_{[0, N-1]} u\right)(0)\left|\left(\Phi_{[0, N-1]} u\right)(1)\right| \cdots \mid\left(\Phi_{[0, N-1]} u\right)(N-1)\right]^{\mathrm{T}} .
\end{aligned}
$$

Then

$$
\begin{aligned}
& \Phi_{[1, N]} y=[y(1)|y(2)| \cdots \mid y(N)]^{\mathrm{T}}, \\
& \Phi_{[0, N-1]} u=[u(0)|u(1)| \cdots \mid u(N-1)]^{\mathrm{T}} .
\end{aligned}
$$

Define the matrix operator for a truncation as

$$
\mathbf{M}\left(\Phi_{[1, N]} w\right) \stackrel{\text { def }}{=} \mathbf{W}=w(1) \mathbf{I}+w(2) \mathbf{S}+\cdots+w(N) \mathbf{S}^{N-1},
$$

where $\Phi_{[1, N]} w=[w(1)|w(2)| \cdots \mid w(N)]^{\mathrm{T}}$.

Proposition $2 \boldsymbol{\varepsilon}_{l}=\mathbf{S}^{l-1} \boldsymbol{\varepsilon}_{1}$ for $l=2,3, \ldots, N$, and $\mathbf{S W}=\mathbf{W S}$. Here $\boldsymbol{\varepsilon}_{l}=\mathbf{S}^{l-1} \boldsymbol{\varepsilon}_{1}$ is assigned as the $(l-1)$ th shift impulse signal.

By making the truncation operator to system (2), a lift-vector form description becomes

$$
\Phi_{[1, N]} y=\mathbf{M}\left(\Phi_{[1, N]} g\right) \Phi_{[0, N-1]} u
$$

Denote the $z$-transform truncations with respect to the sequences $\left\{\left(\Phi_{[1, N]} y\right)(n)\right\}$, $\left\{\left(\Phi_{[0, N-1]} u\right)(n)\right\}$, and $\left\{\left(\Phi_{[1, N]} g\right)(n)\right\}$ as

$$
\left(\Phi_{[1, N]} \hat{y}\right)(z)=y(1)+y(2) z^{-1}+\cdots+y(N) z^{-(N-1)}
$$




$$
\begin{aligned}
& \left(\Phi_{[1, N]} \hat{g}\right)(z)=g(1)+g(2) z^{-1}+\cdots+g(N) z^{-(N-1)}, \\
& \left(\Phi_{[0, N-1]} \hat{u}\right)(z)=u(0)+u(1) z^{-1}+\cdots+u(N-1) z^{-(N-1)} .
\end{aligned}
$$

Proposition 3 The relationship of the z-transform truncations with respect to (2) is as follows:

$$
\begin{aligned}
\left(\Phi_{[1, N]} \hat{y}\right)(z)-\left(\Phi_{[1, N]} \hat{g}\right)(z)\left(\Phi_{[0, N-1]} \hat{u}\right)(z) \\
=-(g(N) u(1)+g(N-1) u(2)+\cdots+g(2) u(N-1)) z^{-N} \\
\quad-(g(N) u(2)+g(N-1) u(3)+\cdots+g(3) u(N-1)) z^{-(N+1)} \\
\quad-\cdots-g(N) u(N-1) z^{-2(N-1)} .
\end{aligned}
$$

Proof

$$
\begin{aligned}
\left(\Phi_{[1, N]} \hat{y}\right)(z)= & y(1)+y(2) z^{-1}+\cdots+y(N) z^{-(N-1)} \\
= & g(1) u(0)+(g(2) u(0)+g(1) u(1)) z^{-1} \\
& +\cdots+(g(N) u(0)+g(N-1) u(1)+\cdots+g(1) u(N-1)) z^{-(N-1)} .
\end{aligned}
$$

Additionally,

$$
\begin{aligned}
&\left(\Phi_{[1, N]} \hat{g}\right)(z)\left(\Phi_{[0, N-1]} \hat{u}\right)(z) \\
&=\left(g(1)+g(2) z^{-1}+\cdots+g(N) z^{-(N-1)}\right)\left(u(0)+u(1) z^{-1}+\cdots+u(N-1) z^{-(N-1)}\right) \\
&= g(1) u(0)+(g(2) u(0)+g(1) u(1)) z^{-1} \\
&+\cdots+(g(N) u(0)+g(N-1) u(1)+\cdots+g(1) u(N-1)) z^{-(N-1)} \\
&+(g(N) u(1)+g(N-1) u(2)+\cdots+g(2) u(N-1)) z^{-N} \\
&+(g(N) u(2)+g(N-1) u(3)+\cdots+g(3) u(N-1)) z^{-(N+1)} \\
&+\cdots+g(N) u(N-1) z^{-2(N-1)} .
\end{aligned}
$$

Taking Eqs. (3) and (4) into account achieves the result.

Remark 1 Proposition 3 exhibits that the time-domain convolution theorem, which is fit for infinite-length sequences, is no longer guaranteed for truncated finite-length sequences. Therefore the spectrum at each frequency for a finite-length sequence computed by the $z$-transform making use of time-domain convolution theorem is only an approximate formulation. The approximation precision depends on the truncation length and the stability of the impulse response. Thus the validity of the existing $z$-transform-based controller design, convergence analysis, and robustness for ILC systems in [28-31] needs to be clarified in a rigorous manner. 


\subsection{Discrete Fourier transform for LDTI SISO systems}

Definition 2 (Discrete Fourier transform [32]) For a finite-length discrete sequence $v=$ $[v(0)|v(1)| \cdots \mid v(N-1)]^{\mathrm{T}}$, its discrete Fourier transform (DFT) is defined as

$$
V(m)=\sum_{n=0}^{N-1} v(n) \mathrm{e}^{-j \frac{2 \pi}{N} m \cdot n}, \quad m=0,1, \ldots, N-1 .
$$

Its inverse discrete Fourier transform (IDFT) takes the form

$$
v(n)=\frac{1}{N} \sum_{m=0}^{N-1} V(m) \mathrm{e}^{j \frac{2 \pi}{N} m \cdot n}, \quad n=0,1, \ldots, N-1 .
$$

For simplicity, denote $s=\mathrm{e}^{j \frac{2 \pi}{N}}$. Then $\mathrm{e}^{j \frac{2 \pi}{N} m n}=s^{m n}$, and formula (5) becomes

$$
V(m)=\sum_{n=0}^{N-1} v(n) s^{-m \cdot n}, \quad m=0,1, \ldots, N-1 .
$$

Analogously, its inverse discrete Fourier transform (IDFT) (6) can be rewritten as

$$
v(n)=\frac{1}{N} \sum_{m=0}^{N-1} V(m) s^{m \cdot n} .
$$

Denote

$$
\begin{aligned}
\boldsymbol{v} & =[v(0)|v(1)| \cdots \mid v(N-1)]^{\mathrm{T}}, \\
\boldsymbol{V} & =[V(0)|V(1)| \cdots \mid V(N-1)]^{\mathrm{T}}, \\
\mathbf{Q} & =\left[\begin{array}{ccccc}
1 & 1 & 1 & \cdots & 1 \\
1 & s^{-1 \cdot 1} & s^{-1 \cdot 2} & \cdots & s^{-1 \cdot(N-1)} \\
1 & s^{-2 \cdot 1} & s^{-2 \cdot 2} & \cdots & s^{-2 \cdot(N-1)} \\
\vdots & \vdots & \vdots & \ddots & \vdots \\
1 & s^{-(N-1) \cdot 1} & s^{-(N-1) \cdot 2} & \cdots & s^{-(N-1) \cdot(N-1)}
\end{array}\right] .
\end{aligned}
$$

Then $\boldsymbol{V}=\mathbf{Q} \boldsymbol{v}$ and, equivalently, $\boldsymbol{v}=\mathbf{Q}^{-1} \boldsymbol{V}$, where

$$
\mathbf{Q}^{-1}=\frac{1}{N}\left[\begin{array}{ccccc}
1 & 1 & 1 & \cdots & 1 \\
1 & s^{1 \cdot 1} & s^{1 \cdot 2} & \cdots & s^{1 \cdot(N-1)} \\
1 & s^{2 \cdot 1} & s^{2 \cdot 2} & \cdots & s^{2 \cdot(N-1)} \\
\vdots & \vdots & \vdots & \ddots & \vdots \\
1 & s^{(N-1) \cdot 1} & s^{(N-1) \cdot 2} & \cdots & s^{(N-1) \cdot(N-1)}
\end{array}\right] \text {. }
$$

It is no difficult to derive the famous Parseval energy formula

$$
\|\boldsymbol{V}\|_{2}^{2}=\sum_{n=0}^{N-1}|v(n)|^{2}=\frac{1}{N} \sum_{m=0}^{N-1}|V(m)|^{2}=\frac{1}{N}\|\boldsymbol{V}\|_{2}^{2} .
$$


Moreover,

$$
|\boldsymbol{V}(N-m)|=|\boldsymbol{V}(-m)|=|\boldsymbol{V}(m)|
$$

We further calculate the DFT for a class of LDTI single-input-single-output (SISO) systems described by

$$
\left\{\begin{array}{l}
\boldsymbol{x}(n+1)=\mathbf{A} \boldsymbol{x}(n)+\mathbf{B} u(n), \\
y(n+1)=\mathbf{C} \boldsymbol{x}(n+1), \\
\boldsymbol{x}(0)=\mathbf{0}, \quad n=0,1, \ldots, N-1 ;
\end{array}\right.
$$

where $N$ is a finite positive integer denoting the total sampling number, $\boldsymbol{x}(n), u(n)$, and $y(n)$ are $p$-dimensional state vector, scalar input, and scalar output, respectively, and $\mathbf{A}, \mathbf{B}$, and $\mathbf{C}$ are matrices of appropriate dimensions.

Let $\boldsymbol{u}^{*}=\boldsymbol{\varepsilon}_{1}=\left[\begin{array}{llll}1 & 0 & \cdots & 0\end{array}\right]^{\mathrm{T}}$ be the impulse signal and stimulate systems (9). Then the output takes the form

$$
\boldsymbol{y}^{*}=\boldsymbol{g}_{1}=\left[g_{1}(1)\left|g_{1}(2)\right| \cdots \mid g_{1}(N)\right]^{\mathrm{T}}=\left[\mathbf{C B}|\mathbf{C A B}| \mathbf{C A}^{2} \mathbf{B}|\cdots| \mathbf{C A}^{N-1} \mathbf{B}\right]^{\mathrm{T}}
$$

where the vector $\boldsymbol{g}_{1}$ is assigned as the impulse response of system (9).

Thus, for any input $\boldsymbol{u}=[u(0)|u(1)| \cdots \mid u(N-1)]^{\mathrm{T}}$, the output of system (9) is expressed as

$$
y(n+1)=\sum_{l=0}^{n} g_{1}(n+1-l) u(l), \quad n=0,1, \ldots, N-1 .
$$

For simplicity, denote $y^{+}(n)=y(n+1)$. Then the DFT of the sequence $y^{+}=[y(1), y(2), \ldots$, $y(N)]^{\mathrm{T}}$ is computed as

$$
\begin{aligned}
Y^{+}(m) & =\sum_{n=0}^{N-1} y^{+}(n) s^{-m \cdot n}=\sum_{n=0}^{N-1}\left(\sum_{q=0}^{n} g_{1}(n+1-q) u(q)\right) s^{-m \cdot n} \\
& =\sum_{n=0}^{N-1}\left(\sum_{q=0}^{n} g_{1}(n+1-q) u(q) s^{-m \cdot n}\right) \\
& =\sum_{q=0}^{N-1}\left(\sum_{n=q}^{N-1} g_{1}(n+1-q) s^{-m n}\right) u(q) \\
& =\sum_{q=0}^{N-1}\left(\sum_{p=n-q=0}^{(N-1)-q} g_{1}(p+1) s^{-m(p+q)}\right) u(q), \quad m=0,1, \ldots, N-1 .
\end{aligned}
$$

In addition, according to DFT formula (5a), the DFTs for the sequences $g_{1}=\left[g_{1}(1), g_{1}(2)\right.$, $\left.\ldots, g_{1}(N)\right]^{\mathrm{T}}$ and $u=[u(0), u(1), \ldots, u(N-1)]^{\mathrm{T}}$ are computed as

$$
G_{1}(m)=\sum_{p=0}^{N-1} g_{1}(p+1) s^{-m \cdot p} \quad \text { and } \quad U(m)=\sum_{q=0}^{N-1} u(q) s^{-m \cdot q}
$$


Then

$$
\begin{aligned}
G_{1}(m) U(m) & =\sum_{p=0}^{N-1} g_{1}(p+1) s^{-m \cdot p} \sum_{q=0}^{N-1} u(q) s^{-m \cdot q} \\
& =\sum_{q=0}^{N-1}\left(\sum_{p=0}^{N-1} g_{1}(p+1) s^{-m \cdot(p+q)}\right) u(q), \quad m=0,1, \ldots, N-1 .
\end{aligned}
$$

Comparing the summation expression on the right side of Eq. (11) with that of (12), we observe that usualy $Y^{+}(m) \neq G_{1}(m) U(m)$ unless $N=+\infty$. This means that the time-domain convolution theorem for the discrete Fourier transform applied to a finite-length sequence is not true either. The expression $G_{1}(m) U(m)$ is only regarded as an approximation of $Y^{+}(m)$. Therefore, the so-called approximate spectrum-based convergence and robustness in [34-41] need to be refined in a rigorous manner.

For an example, in the LDTI SISO system (9), denote the spectral radius of the $p$ th-order state matrix $\mathbf{A}$ as $\rho(\mathbf{A})=\max _{1 \leq i \leq p}\left\{\left|\lambda_{i}\right|\right\}$ with $\lambda_{i}, i=1,2, \ldots, p$, being the eigenvalues of $\mathbf{A}$.

For simplicity, denote by $\tilde{Y}(m)=G_{1}(m) U(m)$ the frequency-domain output approximation of the precise frequency-domain output $Y^{+}(m)$.

Let $\left\|Y^{+}\right\|_{2}=\sqrt{\sum_{m=0}^{N-1}\left|Y^{+}(m)\right|^{2}},\|\tilde{Y}\|_{2}=\sqrt{\sum_{m=0}^{N-1}|\tilde{Y}(m)|^{2}}$, and $\left\|y^{+}\right\|_{2}=\sqrt{\sum_{n=0}^{N-1}\left|y^{+}(n)\right|^{2}}$ be the 2-norms of the frequency-domain output, frequency-domain approximate output, and time-domain output, respectively. From Parseval's energy formula (7) we have $\left\|Y^{+}\right\|_{2}=$ $\sqrt{N}\left\|y^{+}\right\|_{2}$.

For comparison, generate an input sequence $\boldsymbol{u}=[u(0)|u(1)| \cdots \mid u(79)]^{\mathrm{T}}$ with components being uniformly distributed random numbers between 0 and 1 .

Case 1: Let

$$
\mathbf{A}=\left[\begin{array}{ccc}
1.8869 & 0 & 0 \\
1.1507 & 0.9989 & 1 \\
0.0008 & 0.100 & 1
\end{array}\right], \quad \mathbf{B}=\left[\begin{array}{c}
0 \\
0.0097 \\
0.0093
\end{array}\right], \quad \text { and } \quad \mathbf{C}=\left[\begin{array}{lll}
0 & 0 & 1
\end{array}\right]
$$

It is computed that $\rho(\mathbf{A})=1.8869>1$. This means that system (9) is unstable. Figure 1 exhibits the frequency-wise magnitude spectra of the frequency-domain output and its approximation when the truncation length $N=20$, from which we see that $|\tilde{Y}(m)|<\left|Y^{+}(m)\right|$ for frequency orders $m=1,2, \ldots, 19$ but $|\tilde{Y}(0)|>\left|Y^{+}(0)\right|$. Figure 2 displays the energy

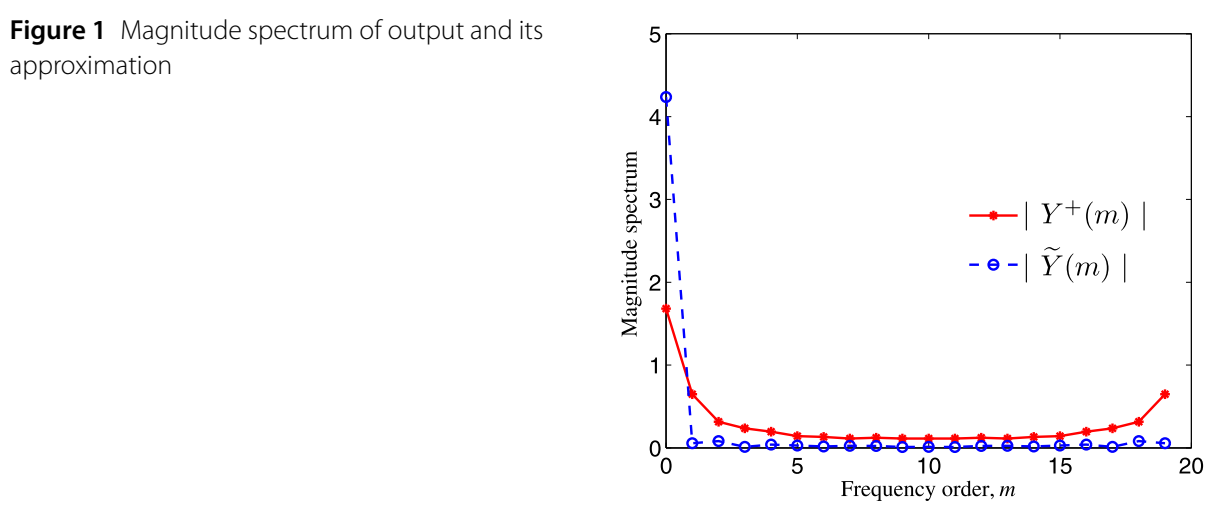


Figure 2 Energy tendency of output and its approximation

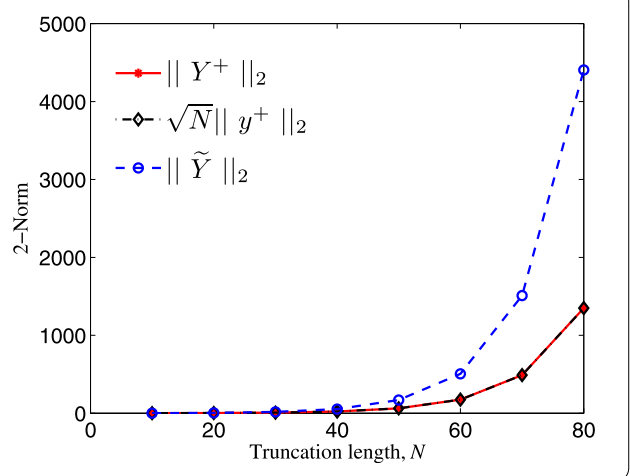

Figure 3 Magnitude spectrum of output and its approximation

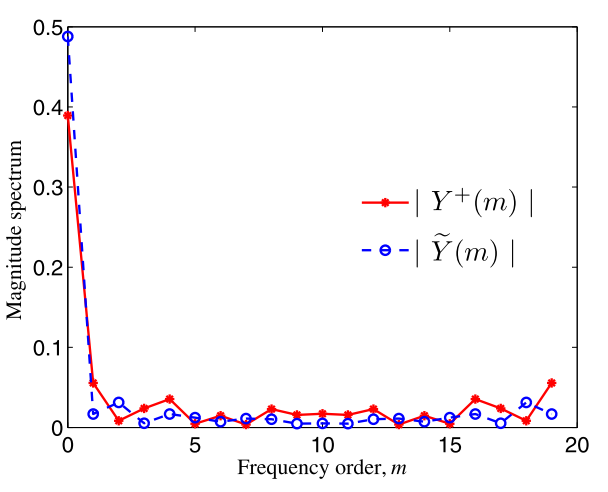

tendencies of the frequency-domain output, frequency-domain approximate output, and time-domain output as the truncation length $N$ increases, which says that the discrepancy of the approximation $\|\tilde{Y}\|_{2}$ from the precise value $\left\|Y^{+}\right\|_{2}$ enlarges as the truncation length $N$ increases.

Case 2: Let

$$
\mathbf{A}=\left[\begin{array}{ccc}
0.8869 & 0 & 0 \\
0.1507 & 0.7989 & 1 \\
0.0008 & 0.100 & 0.7
\end{array}\right], \quad \mathbf{B}=\left[\begin{array}{c}
0 \\
0.0097 \\
0.0093
\end{array}\right], \quad \mathbf{C}=\left[\begin{array}{lll}
0 & 0 & 1
\end{array}\right]
$$

It is testified that $\rho(\mathbf{A})=0.8869<1$. This implies that the system is stable.

Figure 3 depicts the frequency-wise magnitude spectra of the output and its approximation when the truncation length $N=20$, which conveys that the orders of frequency-wise spectra $\left|Y^{+}(m)\right|$ and $|\tilde{Y}(m)|$ are diverse. Whilst Fig. 4 presents the 2-norms of frequencydomain output, frequency-domain approximate output, and time-domain output for the truncation lengths $N=10,20, \ldots, 80$, respectively, which shows that the discrepancy of the approximation $\|\tilde{Y}\|_{2}$ from the precise value $\left\|Y^{+}\right\|_{2}$ does not distinctly enlarge as the truncation length $N$ increases.

Besides, Figs. 1 and 3 deliver that $\left|Y^{+}(m)\right|=\left|Y^{+}(N-m)\right|$ and $|\tilde{Y}(m)|=|\tilde{Y}(N-m)|$ for $m=1,2, \ldots, 19$, whereas Figs. 2 and 4 convey that $\left\|Y^{+}\right\|_{2} \equiv \sqrt{N}\left\|y^{+}\right\|_{2}$.

\subsection{Relationship formulation in discrete-frequency domain}

In what follows, we derive the discrete-frequency relationship among output, shift impulse responses, and input. 
Figure 4 Energy tendency of output and its approximation

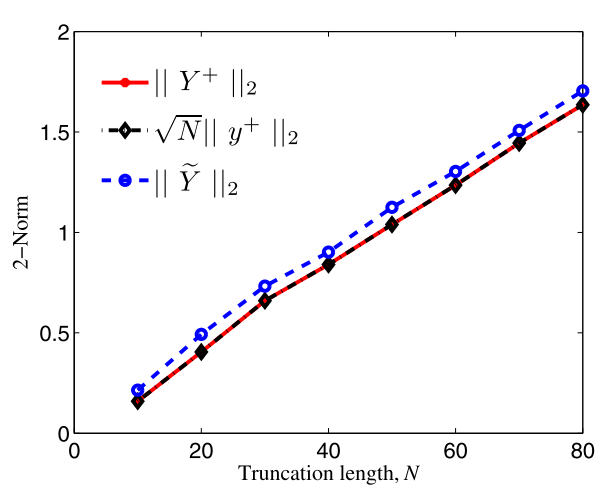

For simplicity, denote

$$
\begin{aligned}
\boldsymbol{y} & =\boldsymbol{y}^{+}=[y(1)|y(2)| \cdots \mid y(N)]^{\mathrm{T}}, \\
\boldsymbol{u} & =[u(0)|u(1)| \cdots \mid u(N-1)]^{\mathrm{T}}, \\
\overline{\mathbf{G}} & =\left[\begin{array}{ccccc}
g_{1}(1) & 0 & 0 & \cdots & 0 \\
g_{1}(2) & g_{1}(1) & 0 & \cdots & 0 \\
g_{1}(3) & g_{1}(2) & g_{1}(1) & \cdots & 0 \\
\vdots & \vdots & \vdots & \ddots & \vdots \\
g_{1}(N) & g_{1}(N-1) & g_{1}(N-2) & \cdots & g_{1}(1)
\end{array}\right] .
\end{aligned}
$$

From (10) we have

$$
\begin{aligned}
\boldsymbol{y} & =\overline{\mathbf{G}} \boldsymbol{u} \\
& =\overline{\mathbf{G}}\left[u(0) \boldsymbol{\varepsilon}_{1}+u(1) \boldsymbol{\varepsilon}_{2}+\cdots+u(N-1) \boldsymbol{\varepsilon}_{N}\right] \\
& =u(0) \overline{\mathbf{G}} \boldsymbol{\varepsilon}_{1}+u(1) \overline{\mathbf{G}} \boldsymbol{\varepsilon}_{2}+\cdots+u(N-1) \overline{\mathbf{G}} \boldsymbol{\varepsilon}_{N} .
\end{aligned}
$$

Denote

$$
\boldsymbol{g}_{l}=\overline{\mathbf{G}} \boldsymbol{\varepsilon}_{l} \quad \text { for } l=1,2, \ldots, N
$$

and

$$
\overline{\mathbf{G}}=\left[\overline{\mathbf{G}}_{1}\left|\overline{\mathbf{G}}_{2}\right| \cdots \mid \overline{\mathbf{G}}_{N}\right]
$$

Then $\overline{\mathbf{G}}_{l}=\boldsymbol{g}_{l}=\overline{\mathbf{G}} \boldsymbol{\varepsilon}_{l}$ for $l=2, \ldots, N$, which means that the $l$ th column of the matrix $\overline{\mathbf{G}}$ is the response of system (10) with respect to the shift impulse $\varepsilon_{l}$, named as the $(l-1)$ th shift impulse response. Hence $\overline{\mathbf{G}}$ is called the impulse response matrix.

Then (10) becomes

$$
\boldsymbol{y}=\overline{\mathbf{G}} \boldsymbol{u}=\left[\boldsymbol{g}_{1}\left|\boldsymbol{g}_{2}\right| \cdots \mid \boldsymbol{g}_{N}\right] \boldsymbol{u}
$$

Therefore

$$
\boldsymbol{Y}=\mathbf{Q} \boldsymbol{y}=\mathbf{Q}\left[\boldsymbol{g}_{1}\left|\boldsymbol{g}_{2}\right| \cdots \mid \boldsymbol{g}_{N}\right] \boldsymbol{u}
$$




$$
\begin{aligned}
& =\left[\mathbf{Q} \boldsymbol{g}_{1}\left|\mathbf{Q} \boldsymbol{g}_{2}\right| \cdots \mid \mathbf{Q} \boldsymbol{g}_{N}\right] \mathbf{Q}^{-1} \mathbf{Q u} \\
& =\left[\boldsymbol{G}_{1}\left|\boldsymbol{G}_{2}\right| \cdots \mid \boldsymbol{G}_{N}\right] \mathbf{Q}^{-1} \boldsymbol{U},
\end{aligned}
$$

where $\boldsymbol{G}_{l}=\left[G_{l}(0)\left|G_{l}(1)\right| \cdots \mid G_{l}(N-1)\right]^{\mathrm{T}}=\mathbf{Q} \boldsymbol{g}_{l}$ for $l=1,2, \ldots, N$, which expresses the DFT of the $(l-1)$ th shift impulse response, and

$$
G_{l}(m)=\sum_{n=0}^{N-1} g_{l}(n+1) s^{-m \cdot n} \quad \text { for } m=0,1,2, \ldots, N-1 .
$$

Denote $\mathbf{G}=\left[\boldsymbol{G}_{1}\left|\boldsymbol{G}_{2}\right| \cdots \mid \boldsymbol{G}_{N}\right]$. Equivalently, Eq. (14) becomes

$$
\boldsymbol{Y}=\mathbf{G Q}^{-1} \mathbf{U}
$$

Equation (16) formulates the discrete-frequency relationship of output, shift impulse responses, and input.

\subsection{Properties of Toeplitz matrix}

Proposition 4 For a lower triangular Toeplitz matrix

$$
\mathbf{M}=\left[\begin{array}{ccccc}
a_{1} & 0 & 0 & \cdots & 0 \\
a_{2} & a_{1} & 0 & \cdots & 0 \\
a_{3} & a_{2} & a_{1} & \cdots & 0 \\
\vdots & \vdots & \vdots & \ddots & \vdots \\
a_{N} & a_{N-1} & a_{N-2} & \cdots & a_{1}
\end{array}\right],
$$

we have $\lim _{k \rightarrow+\infty} \mathbf{M}^{k}=\mathbf{0}$ iff $\lambda=\left|a_{1}\right|<1$.

Proof In view of the expression

$$
\mathbf{M}=a_{1} \mathbf{I}+a_{2} \mathbf{S}+a_{3} \mathbf{S}^{2}+\cdots+a_{N} \mathbf{S}^{N-1},
$$

denote $\mathbf{P}=a_{2}+a_{3} \mathbf{S}+a_{4} \mathbf{S}^{2}+\cdots+a_{N} \mathbf{S}^{N-2}$. Then $\mathbf{P S}=\mathbf{S P}$ and $\mathbf{M}^{k}=\left(a_{1} \mathbf{I}+\mathbf{P S}\right)^{k}$.

Then, for an index $k(k<N)$, Eq. (17) induces

$$
\begin{aligned}
\mathbf{M}^{k}= & \left(a_{1} \mathbf{I}+\mathbf{P S}\right)^{k} \\
= & \left(a_{1}\right)^{k} \mathbf{I}+C_{k}^{1}\left(a_{1}\right)^{k-1} \mathbf{P} \mathbf{S}+\cdots+C_{k}^{l}\left(a_{1}\right)^{k-l} \mathbf{P}^{l} \mathbf{S}^{l} \\
& +\cdots+C_{k}^{k-1}\left(a_{1}\right) \mathbf{P}^{k-1} \mathbf{S}^{k-1}+\mathbf{P}^{k} \mathbf{S}^{k} .
\end{aligned}
$$

For a sufficiently large iteration index $k(k \geq N)$, by considering $\mathbf{S}^{N}=\mathbf{0}$ Eq. (18) results in

$$
\begin{aligned}
\mathbf{M}^{k}= & \left(a_{1} \mathbf{I}+\mathbf{P S}\right)^{k} \\
= & \left(a_{1}\right)^{k} \mathbf{I}+C_{k}^{1}\left(a_{1}\right)^{k-1} \mathbf{P S}+\cdots+C_{k}^{i}\left(a_{1}\right)^{k-l} \mathbf{P}^{l} \mathbf{S}^{l} \\
& +\cdots+C_{k}^{N-1}\left(a_{1}\right)^{k-(N-1)} \mathbf{P}^{N-1} \mathbf{S}^{N-1},
\end{aligned}
$$

where $C_{k}^{l}=\frac{k(k-1) \cdots(k-l+1)}{l(l-1) \cdots 2 \cdot 1}$.

Necessity is obvious. 
Sufficiency: Recall the assumption that $\lambda=\left|a_{1}\right|<1$.

Case 1: For the case where $\lambda=\left|a_{1}\right|=0$, from expression (18) we see that $\mathbf{M}^{N}=\mathbf{0}$.

Case 2: For the case where $0<\lambda=\left|a_{1}\right|<1$, note that

$$
\begin{aligned}
\left|C_{k}^{l}\left(a_{1}\right)^{k-l}\right| & =\frac{k(k-1) \cdots(k-l+1)}{l(l-1) \cdots 2 \cdot 1}\left|a_{1}\right|^{k-l} \\
& =\frac{1}{l(l-1) \cdots 2 \cdot 1} \frac{k^{l}+b_{1} k^{l-1}+\cdots+b_{l-1} k+b_{l}}{\left|\frac{1}{a_{1}}\right|^{k-l}} .
\end{aligned}
$$

By multiple adopting L. Hospital's rule for limiting times, we have

$$
\lim _{k \rightarrow+\infty}\left|C_{k}^{l}\left(a_{1}\right)^{k-l}\right|=\frac{1}{l(l-1) \cdots 2 \cdot 1} \lim _{k \rightarrow+\infty} \frac{l k^{l-1}+(l-1) b_{1} k^{l-2}+\cdots+b_{l-1}}{\left|\frac{1}{a_{1}}\right|^{k-l} \ln \left|\frac{1}{a_{1}}\right|}=\cdots=0 .
$$

As the number $N$ is fixed, Eq. (18) gives rise to $\lim _{k \rightarrow+\infty} \mathbf{M}^{k}=\mathbf{0}$.

Remark 2 It should be pointed out that Proposition 4 can be regarded as a corollary of Schur complementary. However, the derivation of Schur complementary is too complicated to easily acquire the evolution of elements of the Toeplitz matrix to the power of iteration index as the power index $k$ increases. The proof of Proposition 4 is given in a straightforward mode, which explicitly presents the evolution of elements of the Toeplitz matrix to the power of iteration index in a clear way. This is helpful in observing the evolution behavior of the transient learning performance of the iterative learning control system because the transmit matrix of the tracking errors of the two adjacent iterations turns to be a lower triangular Toeplitz matrix.

Proposition 5 If the matrices $\mathbf{M}_{1}$ and $\mathbf{M}_{2}$ are Toeplitz, then $\mathbf{M}_{1} \mathbf{M}_{2}=\mathbf{M}_{2} \mathbf{M}_{1}$ is Toeplitz.

Proof Let $\mathbf{M}_{1}=a_{1} \mathbf{I}+a_{2} \mathbf{S}+a_{3} \mathbf{S}^{2}+\cdots+a_{N} \mathbf{S}^{N-1}$ and $\mathbf{M}_{2}=b_{1} \mathbf{I}+b_{2} \mathbf{S}+b_{3} \mathbf{S}^{2}+\cdots+b_{N} \mathbf{S}^{N-1}$.

Then

$$
\begin{aligned}
\mathbf{M}_{1} \mathbf{M}_{2}= & \left(a_{1} \mathbf{I}+a_{2} \mathbf{S}+a_{3} \mathbf{S}^{2}+\cdots+a_{N} \mathbf{S}^{N-1}\right)\left(b_{1} \mathbf{I}+b_{2} \mathbf{S}+b_{3} \mathbf{S}^{2}+\cdots+b_{N} \mathbf{S}^{N-1}\right) \\
= & a_{1} b_{1} \mathbf{I}+\left(a_{1} b_{2}+a_{2} b_{1}\right) \mathbf{S}+\left(a_{1} b_{3}+a_{2} b_{2}+a_{3} b_{1}\right) \mathbf{S}^{2} \\
& +\cdots+\left(a_{1} b_{m}+a_{2} b_{m-1}+\cdots+a_{m-1} b_{1}\right) \mathbf{S}^{m-1} \\
& +\cdots+\left(a_{1} b_{N}+a_{2} b_{N-1}+\cdots+a_{N} b_{1}\right) \mathbf{S}^{N-1} \\
= & \mathbf{M}_{2} \mathbf{M}_{1} .
\end{aligned}
$$

Proposition 6 For a lower triangular matrix

$$
\mathbf{R}=\left[\begin{array}{ccccc}
a_{11} & 0 & 0 & \cdots & 0 \\
a_{21} & a_{22} & 0 & \cdots & 0 \\
a_{31} & a_{32} & a_{33} & \cdots & 0 \\
\vdots & \vdots & \vdots & \ddots & \vdots \\
a_{N, 1} & a_{N-1,2} & a_{N-2,3} & \cdots & a_{N, N}
\end{array}\right],
$$

we have $\lim _{k \rightarrow+\infty} \mathbf{R}^{k}=\mathbf{0}$ if $a_{1}=\max _{i=1,2, \ldots, N}\left\{\left|a_{i, i}\right|\right\}<1$. 
Proof Let $a_{1}=\max _{i=1,2, \ldots, N}\left\{\left|a_{i, i}\right|\right\}$ and $a_{l+1}=\max _{i=1,2, \ldots, N-l}\left\{\left|a_{i+l, i}\right|\right\}$ for $l=1,2, \ldots, N-1$.

Then

$$
-\mathbf{M} \leq-|\mathbf{R}| \leq \mathbf{R} \leq|\mathbf{R}| \leq \mathbf{M}
$$

where $|\mathbf{A}|=\left(\left|a_{i j}\right|\right) \leq|\mathbf{B}|=\left(\left|b_{i j}\right|\right)$ if and only if $\left|a_{i j}\right| \leq\left|b_{i j}\right|$.

By multiplication of matrices it is no difficult to yield

$$
-\mathbf{M}^{k} \leq \mathbf{R}^{k} \leq \mathbf{M}^{k}
$$

From Proposition 4 we have $\lim _{k \rightarrow+\infty} \mathbf{R}^{k}=\mathbf{0}$.

\section{Convergence analysis}

\subsection{DFT-based convergence for LDTI systems}

\subsubsection{First-order D-type ILC scheme and convergence}

Provided that system (10) attempts to track a predetermined desired trajectory $y_{d}(n+1)$ while it repetitively operates, let $u_{1}(n)$ be an arbitrary initial input, and let $e_{1}(n+1)=y_{d}(n+$ $1)-y_{1}(n+1)$ denote the output error of systems (10) driven by $u_{1}(n), n=0,1,2, \ldots, N-1$.

By compensating $u_{1}(n)$ with its output error $e_{1}(n+1), u_{2}(n)$ is generated. In recursion, the first-order derivative-type iterative learning control (D-type ILC) updating law is formulated as

$$
\begin{aligned}
& u_{1}(n), \text { arbitrary given, } \\
& u_{k+1}(n)=u_{k}(n)+\Gamma e_{k}(n+1), \quad n=0,1,2, \ldots, N-1,
\end{aligned}
$$

where the subscript $k=1,2, \ldots$ denotes the iteration index, and $\Gamma$ is assigned as the derivative learning gain.

Denote

$$
\begin{aligned}
\boldsymbol{y}_{d} & =\left[y_{d}(1)\left|y_{d}(2)\right| \cdots \mid y_{d}(N)\right]^{\mathrm{T}}, \\
\boldsymbol{u}_{k} & =\left[u_{k}(0)\left|u_{k}(1)\right| \cdots \mid u_{k}(N-1)\right]^{\mathrm{T}}, \\
\boldsymbol{y}_{k} & =\left[y_{k}(1)\left|y_{k}(2)\right| \cdots \mid y_{k}(N)\right]^{\mathrm{T}}, \\
\boldsymbol{e}_{k} & =\boldsymbol{y}_{d}-\boldsymbol{y}_{k}=\left[e_{k}(1)\left|e_{k}(2)\right| \cdots \mid e_{k}(N)\right]^{\mathrm{T}}, \\
\boldsymbol{Y}_{k} & =\mathbf{Q} \boldsymbol{y}_{k}, \\
\boldsymbol{E}_{k} & =\mathbf{Q} \boldsymbol{e}_{k}, \\
\boldsymbol{U}_{k} & =\mathbf{Q} \boldsymbol{u}_{k} .
\end{aligned}
$$

Inferring the derivation of Eq. (14), we have

$$
\boldsymbol{y}_{k}=\overline{\mathbf{G}} \boldsymbol{u}_{k} \text {. }
$$

Analogously, the first-order D-type ILC (20) becomes

$$
\boldsymbol{u}_{k+1}=\boldsymbol{u}_{k}+\Gamma \boldsymbol{e}_{k} .
$$


Theorem 1 Assume that for system (10) $g_{1}(1) \neq 0$, and the first-order D-type ILC (22) is applied. Then in the frequency domain, $\lim _{k \rightarrow+\infty} \boldsymbol{E}_{k+1}=\mathbf{0}$ if and only if $\rho_{1}=\left|1-\Gamma g_{1}(1)\right|<1$.

Proof Taking Eqs. (21) and (22) into account yields

$$
\boldsymbol{e}_{k+1}=(\mathbf{I}-\boldsymbol{\Gamma} \overline{\mathbf{G}}) \boldsymbol{e}_{k} .
$$

Then

$$
\boldsymbol{E}_{k+1}=\mathbf{Q} \boldsymbol{e}_{k+1}=\mathbf{Q}(\mathbf{I}-\Gamma \overline{\mathbf{G}}) \mathbf{Q}^{-1} \mathbf{Q} \boldsymbol{e}_{k}=\mathbf{Q}(\mathbf{I}-\Gamma \overline{\mathbf{G}}) \mathbf{Q}^{-1} \boldsymbol{E}_{k} .
$$

Therefore

$$
\boldsymbol{E}_{k+1}=\mathbf{Q}(\mathbf{I}-\Gamma \overline{\mathbf{G}}) \mathbf{Q}^{-1} \boldsymbol{E}_{k}=\mathbf{Q}(\mathbf{I}-\Gamma \overline{\mathbf{G}})^{2} \mathbf{Q}^{-1} \boldsymbol{E}_{k-1}=\cdots=\mathbf{Q}(\mathbf{I}-\Gamma \overline{\mathbf{G}})^{k} \mathbf{Q}^{-1} \boldsymbol{E}_{1} .
$$

In Eqs. (23) and (24),

$$
\mathbf{I}-\Gamma \overline{\mathbf{G}}=\left[\begin{array}{ccccc}
1-\Gamma g_{1}(1) & 0 & 0 & \cdots & 0 \\
-\Gamma g_{1}(2) & 1-\Gamma g_{1}(1) & 0 & \cdots & 0 \\
-\Gamma g_{1}(3) & -\Gamma g_{1}(2) & 1-\Gamma g_{1}(1) & \cdots & 0 \\
\vdots & \vdots & \vdots & \ddots & \vdots \\
-\Gamma g_{1}(N) & -\Gamma g_{1}(N-1) & -\Gamma g_{1}(N-2) & \cdots & 1-\Gamma g_{1}(1)
\end{array}\right] .
$$

Noting that the matrix $\mathbf{I}-\Gamma \overline{\mathbf{G}}$ is lower triangular Toeplitz, from Proposition 4 the sufficiency and necessity are immediate.

Remark 3 Because the matrix $\mathbf{Q}$ is invertible, $\lim _{k \rightarrow+\infty} \boldsymbol{E}_{k+1}=\mathbf{0}$ in the discrete-frequency domain if and only if $\lim _{k \rightarrow+\infty} \boldsymbol{e}_{k+1}=\mathbf{0}$ in the discrete-time domain. This is theoretically logic and thus convincing. Thus the robustness of the ILC algorithm to the system parameters uncertainty may be addressed in the time domain in a direct manner. This issue is involved in our future work. As for the existing frequency-domain convergence analysis by means of the $z$-transform, the convergence equivalence to the discrete-domain result is quite obscure, the robustness analysis such as of the system parameter uncertainty by the $z$-transform needs to be refined in a rigorous manner, as mentioned in Remark 1.

Remark 4 Though the frequency-domain relationship of (16) is not simpler than the relationship in the time domain, Theorem 1 of the paper conveys that the lifted tracking error vector converges itself. This means that the tracking error may converge while measured in any form of norm. This is benefited from the property of the lower triangular Toeplitz matrix addressed by Proposition 4. However, in many existing convergence results, the discrete-time convergence of the traditional D-type iterative learning control algorithm is ensured in the sense of the lifted tracking error measured in some of but not a preferred norm.

Remark 5 The derivation of Theorem 1 conveys that the convergence condition $\rho_{1}=\mid 1-$ $\Gamma g_{1}(1) \mid<1$ depends upon the systems input matrix $\mathbf{B}$, output matrix $\mathbf{C}$, and the derivative 
learning gain $\Gamma$, but no relevance to the system matrix A. This coincides with the existing conclusion in the literature [31].

Remark 6 From the proof of Lemma 1 we found that the magnitude of tracking error would grow extremely at the first finite iterations. This makes the use of ILC cautious for practical applications. A preferred candidate guarantees the ILC algorithm (22) to be monotonously convergent.

Theorem 2 Assume that the D-type ILC (22) is applied to system (10) and satisfies the condition $\tilde{\rho}_{1}=\|\mathbf{I}-\Gamma \overline{\mathbf{G}}\|_{2}<1$. Then $\left\|\boldsymbol{E}_{k+1}\right\|_{2}<\left\|\boldsymbol{E}_{k}\right\|_{2}$ and $\lim _{k \rightarrow+\infty}\left\|\boldsymbol{E}_{k+1}\right\|_{2}=0$.

Proof Calculating the 2-norm of both sides of expression (23), we have

$$
\left\|\boldsymbol{e}_{k+1}\right\|_{2} \leq\|\mathbf{I}-\Gamma \overline{\mathbf{G}}\|_{2}\left\|\boldsymbol{e}_{k}\right\|_{2}
$$

From Parseval's energy formula (7), (27a) is equivalently reformed as

$$
\left\|\boldsymbol{E}_{k+1}\right\|_{2} \leq\|\mathbf{I}-\Gamma \overline{\mathbf{G}}\|_{2}\left\|\boldsymbol{E}_{k}\right\|_{2}
$$

According to the 2-norm of matrix $\mathbf{I}-\Gamma \overline{\mathbf{G}}$,

$$
\|\mathbf{I}-\Gamma \overline{\mathbf{G}}\|_{2}=\sqrt{\rho\left((\mathbf{I}-\Gamma \overline{\mathbf{G}})^{\mathrm{T}}(\mathbf{I}-\Gamma \overline{\mathbf{G}})\right)},
$$

where $\rho\left((\mathbf{I}-\Gamma \overline{\mathbf{G}})^{\mathrm{T}}(\mathbf{I}-\Gamma \overline{\mathbf{G}})\right)=\max _{i}\left(\lambda_{i}\left((\mathbf{I}-\Gamma \overline{\mathbf{G}})^{\mathrm{T}}(\mathbf{I}-\Gamma \overline{\mathbf{G}})\right)\right)$ denotes the spectral radius of the matrix $(\mathbf{I}-\Gamma \overline{\mathbf{G}})^{\mathrm{T}}(\mathbf{I}-\Gamma \overline{\mathbf{G}})$.

Considering the assumption $\tilde{\rho}_{1}=\|\mathbf{I}-\Gamma \overline{\mathbf{G}}\|_{2}<1$, the results are ensured.

Remark 7 From the above-mentioned convergence condition, the choice of learning gain $\Gamma$ is one degree of freedom but depends upon the impulse response at all sampling instants. This makes the choice of learning gain $\Gamma$ difficult. For the regard, a possible manner is to adaptively construct a time-varying iteration-dependent ILC algorithm.

\subsection{DFT-based convergence for LDTV systems}

Consider the class of LDTV systems described as

$$
\left\{\begin{array}{l}
\boldsymbol{x}(n+1)=\mathbf{A}(n) \mathbf{x}(n)+\mathbf{B}(n) u(n) \\
y(n+1)=\mathbf{C}(n+1) \mathbf{x}(n+1) \\
\mathbf{x}(0)=\mathbf{0}, \quad n=0,1, \ldots, N-1
\end{array}\right.
$$

where $N$ is the total sampling number, $\boldsymbol{x}(n), u(n)$, and $y(n)$ are $p$-dimensional state vector, scalar input, and output, respectively, and $\mathbf{A}(n), \mathbf{B}(n)$, and $\mathbf{C}(n)$ are time-varying matrices of appropriate dimensions.

Let $\boldsymbol{u}^{*}=\boldsymbol{\varepsilon}_{1}=\left[\begin{array}{llll}1 & 0 & \cdots & 0\end{array}\right]^{\mathrm{T}}$ be the impulse signal and stimulate systems (28). Then the output takes the form

$$
\boldsymbol{y}^{*}=\tilde{g}_{1}=\left[\tilde{g}_{1}(1)\left|\tilde{g}_{1}(2)\right| \cdots \mid \tilde{g}_{1}(N)\right]^{\mathrm{T}},
$$


where $\tilde{g}_{1}(1)=\mathbf{C}(1) \mathbf{B}(0)$ and $\tilde{g}_{1}(l)=\mathbf{C}(l)\left(\prod_{i=1}^{l-1} \mathbf{A}(i)\right) \mathbf{B}(0)$ for $l=2,3, \ldots, N$. The vector $\tilde{g}_{1}$ is assigned as the impulse response of LDTV system (28).

Thus, for any input $\boldsymbol{u}=[u(0)|u(1)| \cdots \mid u(N-1)]^{\mathrm{T}}$, its output is expressed as

$$
y(n+1)=\sum_{l=0}^{n} \tilde{g}_{l+1}(n+1-l) u(l), \quad n=0,1, \ldots, N-1,
$$

where $\tilde{g}_{m}(1)=\mathbf{C}(m) \mathbf{B}(m-1)$ and $\tilde{g}_{m}(l)=\mathbf{C}(m+l-1)\left(\prod_{i=1}^{m+l-2} \mathbf{A}(i)\right) \mathbf{B}(m-1)$ for $m=$ $1,2, \ldots, N$ and $l=2,3, \ldots, N+1-m$.

Next, we derive a discrete-frequency relationship among output, shift impulse responses, and input for LDTV system (28).

Denote

$$
\begin{aligned}
\boldsymbol{y} & =[y(1)|y(2)| \cdots \mid y(N)]^{\mathrm{T}}, \\
\boldsymbol{u} & =[u(0)|u(1)| \cdots \mid u(N-1)]^{\mathrm{T}}, \\
\widetilde{\mathbf{H}} & =\left[\begin{array}{ccccc}
\tilde{g}_{1}(1) & 0 & 0 & \cdots & 0 \\
\tilde{g}_{1}(2) & \tilde{g}_{2}(1) & 0 & \cdots & 0 \\
\tilde{g}_{1}(3) & \tilde{g}_{2}(2) & \tilde{g}_{3}(1) & \cdots & 0 \\
\vdots & \vdots & \vdots & \ddots & \vdots \\
\tilde{g}_{1}(N) & \tilde{g}_{2}(N-1) & \tilde{g}_{3}(N-2) & \cdots & \tilde{g}_{N}(1)
\end{array}\right] .
\end{aligned}
$$

From (29) we have

$$
\begin{aligned}
\boldsymbol{y} & =\widetilde{\mathbf{H}} \boldsymbol{u} \\
& =\widetilde{\mathbf{H}}\left[u(0) \boldsymbol{\varepsilon}_{1}+u(1) \boldsymbol{\varepsilon}_{2}+\cdots+u(N-1) \boldsymbol{\varepsilon}_{N}\right] \\
& =u(0) \widetilde{\mathbf{H}} \boldsymbol{\varepsilon}_{1}+u(1) \widetilde{\mathbf{H}} \boldsymbol{\varepsilon}_{2}+\cdots+u(N-1) \widetilde{\mathbf{H}} \boldsymbol{\varepsilon}_{N} .
\end{aligned}
$$

Denote

$$
\tilde{\boldsymbol{g}}_{l}=\widetilde{\mathbf{H}} \boldsymbol{\varepsilon}_{l} \quad \text { for } l=1,2, \ldots, N
$$

and

$$
\widetilde{\mathbf{H}}=\left[\widetilde{\boldsymbol{H}}_{1}\left|\widetilde{\boldsymbol{H}}_{2}\right| \widetilde{\boldsymbol{H}}_{3}|\cdots| \widetilde{\boldsymbol{H}}_{N}\right] .
$$

Then $\widetilde{\boldsymbol{H}}_{l}=\tilde{\boldsymbol{g}}_{l}=\widetilde{\mathbf{H}} \boldsymbol{\varepsilon}_{l}$ for $l=2, \ldots, N$, which means that the $l$ th column of the matrix $\widetilde{\mathbf{H}}$ is the response of system (29) with respect to the shift impulse $\varepsilon_{l}$, called the $(l-1)$ th shift impulse response. Then (29) becomes

$$
\boldsymbol{y}=\widetilde{\mathbf{H}} \boldsymbol{u}=\left[\tilde{\boldsymbol{g}}_{1}\left|\tilde{\boldsymbol{g}}_{2}\right| \cdots \mid \tilde{\boldsymbol{g}}_{N}\right] \boldsymbol{u}
$$

Therefore

$$
\boldsymbol{Y}=\mathbf{Q} \boldsymbol{y}=\mathbf{Q}\left[\tilde{\boldsymbol{g}}_{1}\left|\tilde{\boldsymbol{g}}_{2}\right| \cdots \mid \tilde{\boldsymbol{g}}_{N}\right] \boldsymbol{u}
$$




$$
\begin{aligned}
& =\left[\mathbf{Q} \tilde{\boldsymbol{g}}_{1}\left|\mathbf{Q} \tilde{\boldsymbol{g}}_{2}\right| \cdots \mid \mathbf{Q} \tilde{\boldsymbol{g}}_{N}\right] \mathbf{Q}^{-1} \mathbf{Q u} \\
& =\left[\widetilde{\boldsymbol{G}}_{1}\left|\widetilde{\boldsymbol{G}}_{2}\right| \cdots \mid \widetilde{\mathbf{G}}_{N}\right] \mathbf{Q}^{-1} \boldsymbol{U},
\end{aligned}
$$

where $\widetilde{\mathbf{G}}_{l}=\left[\widetilde{G}_{l}(0)\left|\widetilde{G}_{l}(1)\right| \cdots \mid \widetilde{G}_{l}(N-1)\right]^{\mathrm{T}}=\mathbf{Q} \tilde{\boldsymbol{g}}_{l}$ for $l=1,2, \ldots, N$, which expresses the DFT of the $(l-1)$ th shift impulse response, and

$$
\widetilde{G}_{l}(m)=\sum_{n=0}^{N-1} \tilde{g}_{l}(n+1) s^{-m \cdot n} \quad \text { for } m=0,1,2, \ldots, N-1
$$

Denote $\widetilde{\mathbf{G}}=\left[\widetilde{\mathbf{G}}_{1}\left|\widetilde{\mathbf{G}}_{2}\right| \cdots \mid \widetilde{\mathbf{G}}_{N}\right]$. Equivalently, Eq. (31) becomes

$$
\boldsymbol{Y}=\widetilde{\mathbf{G}} \mathbf{Q}^{-1} \boldsymbol{U}
$$

Equation (33) formulates the discrete-frequency relationship of output, shift impulse responses, and input.

Comparing the input-output equation (33) for LDTV system (28) with the input-output equation (16) for LDTI system (10), the constructive forms of the input-output transmit matrices are identical, except that the elements $\widetilde{G}_{l}(m)$ expressed by $(32)$ are different from $G_{l}(m)$ given by (15). By considering the difference and using Proposition 6, we analogously achieve the following convergence results.

Theorem 3 Assume that, for system (28), $\tilde{g}_{l}(1) \neq 0, l=1,2, \ldots, N$, and the first-order $D$ type ILC (22) is applied. Then, in the frequency domain, $\lim _{k \rightarrow+\infty} \boldsymbol{E}_{k+1}=\mathbf{0}$ if and only if $\rho_{2}=\max _{l=1,2, \ldots, N}\left|1-\Gamma \tilde{g}_{l}(1)\right|<1$.

Remark 8 Theorem 3 reveals that the convergence is guaranteed in the vector form either for the time-domain tracking error or for the frequency-domain tracking error. This implies that the tracking error converges in any form of norm. However, the existing results are only for a fixed but unknown norm [26, 27], although they are equivalent.

Theorem 4 Assume that the D-type ILC (22) is applied to system (28) and satisfies the condition $\tilde{\rho}_{2}=\|\mathbf{I}-\Gamma \tilde{\mathbf{H}}\|_{2}<1$. Then $\left\|\boldsymbol{E}_{k+1}\right\|_{2}<\left\|\boldsymbol{E}_{k}\right\|_{2}$ and $\lim _{k \rightarrow+\infty}\left\|\boldsymbol{E}_{k+1}\right\|_{2}=0$.

Remark 9 Theorem 4 obtains frequency-domain sufficient conditions for the monotonic convergence of a class of LDTV D-type ILC systems, whilst the $z$-transform-based frequency analysis is very hard to compute the tracking error spectrum either for the infinitelength or for the finite-length time-varying system. The result is marvelous for practical applications.

\section{Numerical simulations}

Example 1 Consider the LDTI SISO system

$$
\left\{\begin{array}{l}
{\left[\begin{array}{l}
x_{k}^{1}(n+1) \\
x_{k}^{2}(n+1) \\
x_{k}^{3}(n+1)
\end{array}\right]=\left[\begin{array}{ccc}
1 & 0.02 & 0 \\
0 & 1 & 0.02 \\
0 & -0.0009 & 0.9922
\end{array}\right]\left[\begin{array}{l}
x_{k}^{1}(n) \\
x_{k}^{2}(n) \\
x_{k}^{3}(n)
\end{array}\right]+\left[\begin{array}{c}
0 \\
0 \\
0.02
\end{array}\right] u_{k}(n),} \\
y_{k}(n)=\left[\begin{array}{ll}
0.0018 & 0.02720 .0905
\end{array}\right]\left[\begin{array}{l}
x_{k}^{1}(n) \\
x_{k}^{2}(n) \\
x_{k}^{3}(n)
\end{array}\right], \quad n \in \overline{\mathbf{D}} .
\end{array}\right.
$$


Figure 5 Tracking behavior of the D-type ILC

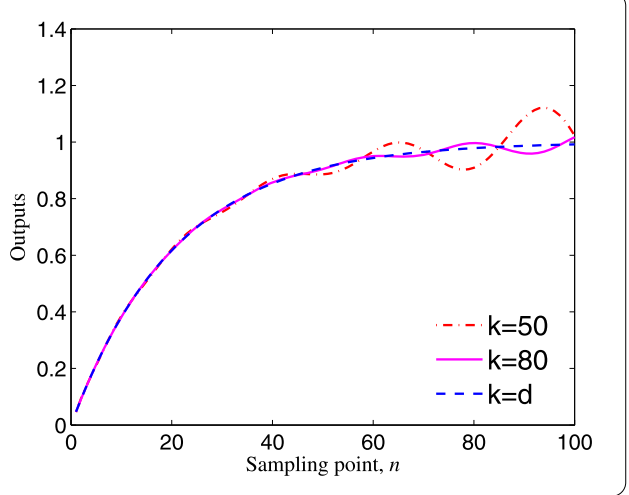

Figure 6 Tracking error tendency in discrete-time domain

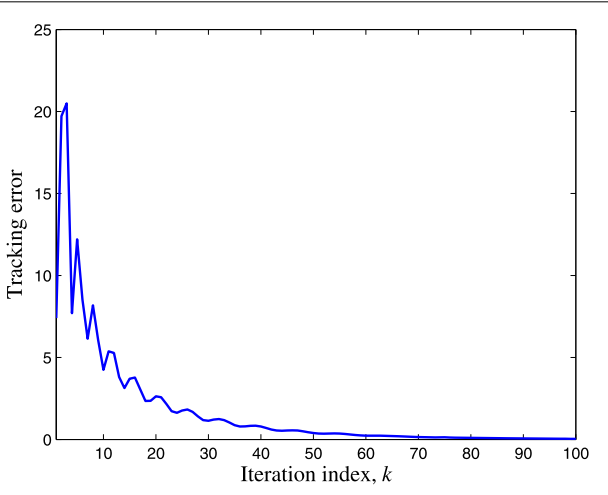

The sampling number of system (34) is set as $\overline{\mathbf{D}}=\{0,1,2, \ldots, 99\}$. The desired trajectory is chosen as $y_{d}(n)=1-\exp (-0.048 n), n \in \overline{\mathbf{D}}$, and the beginning control input is chosen as $u_{1}(n)=1, n \in \overline{\mathbf{D}}$. The initial state is set as $\boldsymbol{x}_{k}(0)=\left[x_{k}^{1}(0), x_{k}^{2}(0), x_{k}^{3}(0)\right]^{\mathrm{T}}=[0,0,0]^{\mathrm{T}}$. It is obvious that $e_{k}(0)=0$. For the D-type ILC algorithm (22), we choose the derivative learning gain $\Gamma=40$. It is computed that $\rho_{1}=\left|1-\Gamma g_{1}(1)\right|=0.9276$, which means that the convergent condition $\rho_{1}<1$ holds.

Figure 5 exhibits the tracking behavior of system (34) driven by the D-type ILC (22), where the dash curve stands for the desired trajectory, and the dash-dotted and solid ones are the outputs at the fiftieth and eightieth iterations, respectively, which shows that the output tracks the desired trajectory closer as the iteration goes on. Figure 6 depicts the tracking error tendency along iteration direction in the sense of 2-norm of $\left\|\boldsymbol{e}_{k}\right\|_{2}=\sqrt{\sum_{n=1}^{100}\left|e_{k}(n)\right|^{2}}$ in the discrete-time domain.

Figure 7 exhibits the frequency-wise spectra of the tracking error at the fiftieth, eightieth, and hundredth iterations, respectively. Figure 8 displays the tracking error energy tendency expressed by $\left\|\boldsymbol{E}_{k}\right\|_{2}=\sqrt{\sum_{m=0}^{99}\left|E_{k}(m)\right|^{2}}$.

Example 2 Consider the other LDTI SISO system

$$
\left\{\begin{array}{l}
{\left[\begin{array}{l}
x_{k}^{1}(n+1) \\
x_{k}^{2}(n+1)
\end{array}\right]=\left[\begin{array}{cc}
1 & 0.02 \\
-0.04 & 0.94
\end{array}\right]\left[\begin{array}{l}
x_{k}^{1}(n) \\
x_{k}^{2}(n)
\end{array}\right]+\left[\begin{array}{c}
0 \\
0.02
\end{array}\right] u_{k}(n),} \\
y_{k}(n)=\left[\begin{array}{ll}
0 & 1
\end{array}\right]\left[\begin{array}{l}
x_{k}^{1}(n) \\
x_{k}^{2}(n)
\end{array}\right], \quad n \in \overline{\mathbf{D}} .
\end{array}\right.
$$


Figure 7 Frequency-wise error spectra

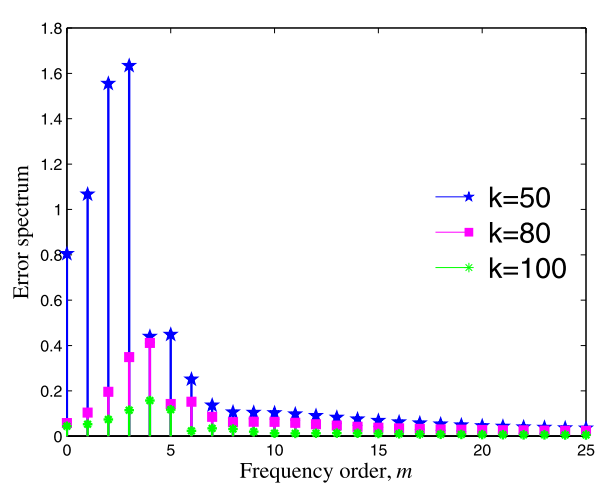

Figure 8 Tracking error tendency in frequency domain

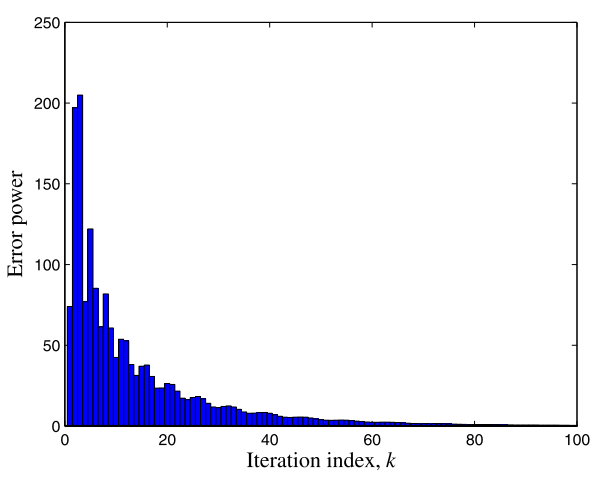

Figure 9 Tracking behavior of the D-type ILC

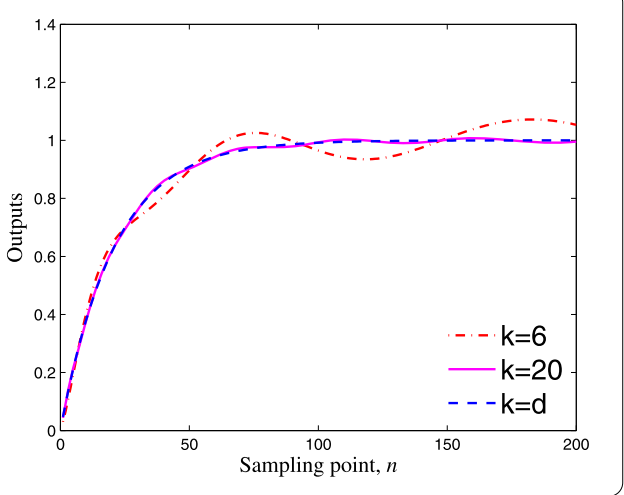

The sampling time of system (35) is set as $\overline{\boldsymbol{D}}=\{0,1,2, \ldots, 199\}$. The desired trajectory is given as $y_{d}(n)=1-\exp (-0.048 n), n \in \overline{\mathbf{D}}$, and the beginning control input is fixed as $u_{1}(n)=1, n \in \overline{\mathbf{D}}$. The initial state is set as $\boldsymbol{x}_{k}(0)=\left[x_{k}^{1}(0), x_{k}^{2}(0)\right]^{\mathrm{T}}=[0,0]^{\mathrm{T}}$. It is obvious that $e_{k}(0)=0$. For the D-type ILC algorithm (22), we select the derivative learning gain $\Gamma=5.6$. It is computed that $\tilde{\rho}_{1}=\|\mathbf{I}-\Gamma \overline{\mathbf{G}}\|_{2}=0.9423$, which means that the monotone convergence condition $\tilde{\rho}_{1}<1$ holds.

Figure 9 exhibits the tracking behavior of the system (35) driven by the D-type ILC (22), where the dash curve stands for the desired trajectory, and the dash-dotted and solid ones are the outputs at the sixth and twentieth iterations, respectively, which shows that the output tracks the desired trajectory more and more closer as the iteration goes on. Fig- 
Figure 10 Tracking error tendency in discrete-time domain

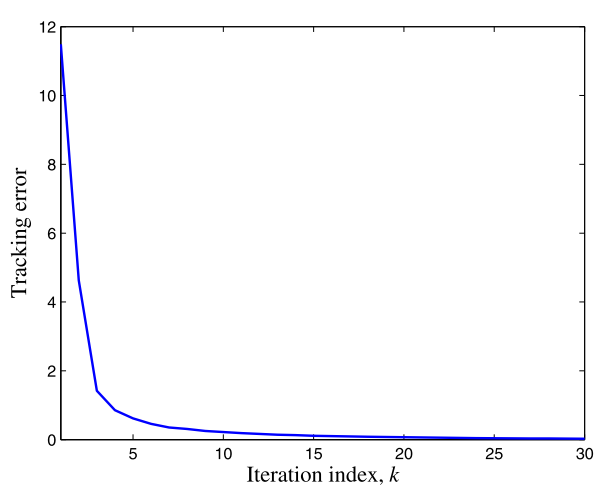

Figure 11 Frequency-wise error spectra

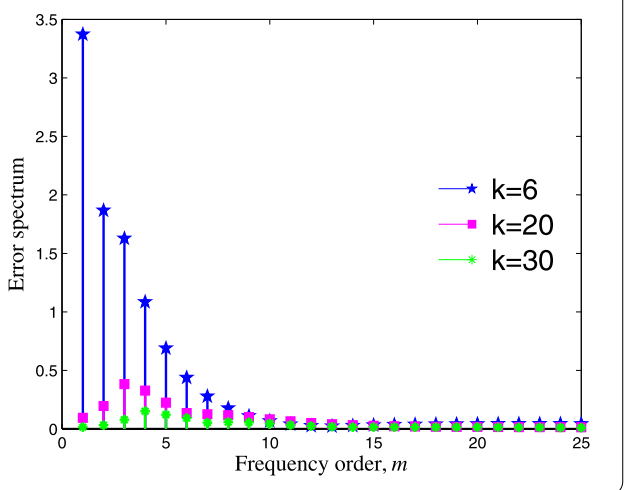

Figure 12 Tracking error tendency in frequency domain

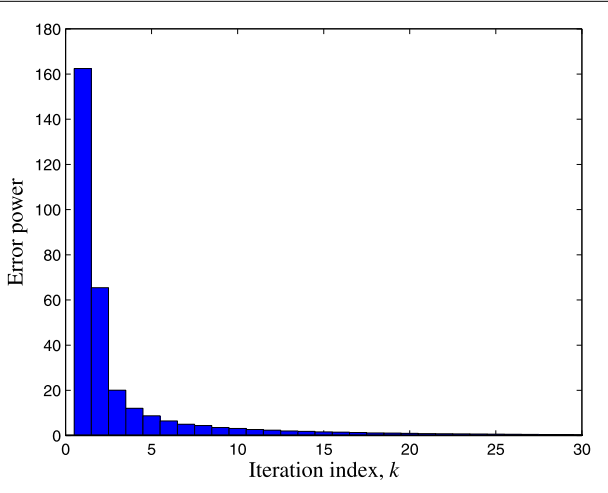

ure 10 depicts the monotone convergence of the tracking error $\left\|\boldsymbol{e}_{k}\right\|_{2}=\sqrt{\sum_{n=1}^{200}\left|e_{k}(n)\right|^{2}}$ produced by the D-type ILC (22) in the discrete-time domain.

Figure 11 exhibits the frequency-wise spectra of the tracking error at the sixth, twentieth, and thirtieth iterations, respectively, which shows that, for each harmonic frequency $\frac{2 \pi}{200} m$, the spectrum $\left|E_{k}(m)\right|$ is decreasing as the iteration index $k$ increases. Figure 12 displays the monotone convergence of the tracking error power $\left\|\boldsymbol{E}_{k}\right\|_{2}=\sqrt{\sum_{m=0}^{199}\left|E_{k}(m)\right|^{2}}$ in the discrete-frequency domain.

\section{Conclusion}

The paper firstly exploits DFT properties of iterative learning control systems and the property of a Toeplitz matrix. The exploitation exhibits that, for repetitive finite-length 
iterative learning control systems, the time-domain convolution theorem either for the $z$ transform or for the DFT form is no longer true by rigorous reduction. This challenges the existing methodologies and results in deed. Though the derived DFT-based frequencydomain relationship among the input, impulse response, and the output looks complicated, it objectively reveals their inherent features, and thus it is not difficult to achieve the equivalence of the convergence in the frequency domain to the existing results in the time domain. Besides, the adopted DFT-based frequency technique is feasible for linear discrete time-variable systems. This will greatly extend the applicable scope. In addition, the convergences for LDTI systems and for LDTV systems have been made in a straightforward manner by means of investigating the convergence property of a Toeplitz matrix to the power of iteration index. This is important for an ILC system to present its tracking behavior evolution along the iteration direction. However, this paper does not involve the frequency-domain robustness to system uncertainties and external noise perturbations. This will be addressed in future works.

Acknowledgements

The authors sincerely thank the referees for their suggestions and comments.

Funding

This work was supported by the National Natural Science Foundation of China (No. F010114-61273135).

Competing interests

The authors declare that they have no competing interests.

Authors' contributions

Both authors contributed equally and significantly in writing this paper. Both authors read and approved the final manuscript.

\section{Publisher's Note}

Springer Nature remains neutral with regard to jurisdictional claims in published maps and institutional affiliations.

Received: 22 September 2018 Accepted: 29 January 2019 Published online: 12 February 2019

References

1. Uchiyama, M.: Formulation of high-speed motion pattern of a mechanical arm by trial. Trans. Soc. Instrum. Control Eng. 14(6), 706-712 (1978)

2. Arimoto, S., Kawamura, S., Miyazaki, F.: Bettering operation of robots by learning. J. Robot. Syst. 1(2), 123-140 (1984)

3. Arimoto, S., Kawamura, S., Miyazaki, F., Tamaki, S.: Learning control theory for dynamical systems. In: Proc. 24th IEEE Conf. Decis. Control, vol. 24, pp. 1375-1380 (1985)

4. Bristow, D.A., Tharayil, M., Alleyne, A.G.: A survey of iterative learning control. IEEE Control Syst. Mag. 26(3), 96-114 (2006)

5. Moore, K.L., Chen, Y., Ahn, H.S.: Iterative learning control: a tutorial and big picture view. In: Proc. 45th IEEE Conf. Decis. Control, vol. 45, pp. 2352-2357 (2006)

6. Ahn, H.S., Chen, Y.Q., Moore, K.L.: Iterative learning control: brief survey and categorization. IEEE Trans. Syst. Man Cybern., Part C, Appl. Rev. 37(6), 1099-1121 (2007)

7. Wang, Y., Gao, F., Doyle, F.J.: Survey on iterative learning control, repetitive control, and run-to-run control. J. Process Control 19(10), 1589-1600 (2009)

8. Xu, J.X.: A survey on iterative learning control for nonlinear systems. Int. J. Control 84(7), 1275-1294 (2011)

9. Ruan, X.E., Park, K.H., Bien, Z.Z:: Retrospective review of some iterative learning control techniques with a comment on prospective long-term learning. Control Theory Appl. 29(8), 966-973 (2012)

10. Shen, D., Wang, Y.: Survey on stochastic iterative learning control. J. Process Control 24(12), 64-77 (2014)

11. Nageshrao, S.P., Lopes, G.A.D., Jeltsema, D., Babuška, R.: Port-Hamiltonian systems in adaptive and learning control: a survey. IEEE Trans. Autom. Control 61(5), 1223-1238 (2015)

12. Shen, D.: Iterative learning control with incomplete information: a survey. IEEE/CAA J. Autom. Sin. 5(5), 885-901 (2018)

13. Bu, X., Hou, Z., Hou, Z., Yang, J.: Robust iterative learning control design for linear systems with time-varying delays and packet dropouts. Adv. Differ. Equ. 2017(1), 84 (2017)

14. Norrlöf, M., Gunnarsson, S.: Time and frequency domain convergence properties in iterative learning control. Int. J. Control 75(14), 1114-1126 (2002)

15. Amann, N., Owens, D.H., Rogers, E.: Iterative learning control for discrete-time systems with exponential rate of convergence. IEE Proc., Control Theory Appl. 143(2), 217-224 (1996) 
16. Jeong, G.M., Choi, C.H.: Iterative learning control for linear discrete time nonminimum phase systems. Automatica 38(2), 287-291 (2002)

17. Moore, K.L., Chen, Y.Q., Bahl, V.: Monotonically convergent iterative learning control for linear discrete-time systems. Automatica 41(9), 1529-1537 (2005)

18. Tian, S., Liu, Q., Dai, X., Zhang, J.: A PD-type iterative learning control algorithm for singular discrete systems. Adv. Differ. Equ. 2016(1), 321 (2016)

19. Li, X.D., Ho, J.K.L., Chow, T.W.S.: Iterative learning control for linear time-variant discrete systems based on 2-D system theory. IEE Proc., Control Theory Appl. 152(1), 13-18 (2005)

20. Liu, Y., Jia, Y: Robust formation control of discrete-time multi-agent systems by iterative learning approach. Int. J. Syst. Sci. 46(4), 625-633 (2015)

21. Zhu, Q., Hu, G.D., Liu, W.Q.: Iterative learning control design method for linear discrete-time uncertain systems with iteratively periodic factors. IET Control Theory Appl. 9(15), 2305-2311 (2015)

22. Ding, J., Cichy, B., Galkowski, K., Rogers, E., Yang, H.Z.: Robust fault-tolerant iterative learning control for discrete systems via linear repetitive processes theory. Int. J. Autom. Comput. 12(3), 254-265 (2015)

23. $\mathrm{Xu}, \mathrm{Y} .$, Shen, $\mathrm{D} ., \mathrm{Bu}, \mathrm{X}:$ Zero-error convergence of iterative learning control using quantized error information. IMA J. Math. Control Inf. 34(3), 1061-1077 (2016)

24. Liu, J., Ruan, X.: Networked iterative learning control for discrete-time systems with stochastic packet dropouts in input and output channels. Adv. Differ. Equ. 2017(1), 53 (2017)

25. Liang, C., Wang, J., Shen, D.: Iterative learning control for linear discrete delay systems via discrete matrix delayed exponential function approach. J. Differ. Equ. Appl. 24(11), 1756-1776 (2018)

26. Yang, S., Xu, J.X., Huang, D., Tan, Y.: Optimal iterative learning control design for multi-agent systems consensus tracking. Syst. Control Lett. 69(2), 80-89 (2014)

27. Bu, X., Hou, Z., Cui, L., Yang, J.: Stability analysis of quantized iterative learning control systems using lifting representation. Int. J. Adapt. Control Signal Process. 31(9), 1327-1336 (2017)

28. Gunnarsson, S., Norrlöf, M.: On the design of ILC algorithms using optimization. Automatica 37(12), 2011-2016 (2001)

29. Owens, D.H., Hatonen, J.J., Daley, S.: Robust monotone gradient-based discrete-time iterative learning control. Int. J. Robust Nonlinear Control 19(6), 634-661 (2009)

30. Paszke, W., Rogers, E., Gałkowski, K.: Experimentally verified generalized KYP lemma based iterative learning control design. Control Eng. Pract. 53, 57-67 (2016)

31. Hatonen, J.J., Moore, K.L., Owens, D.H.: An algebraic approach to iterative learning control. Int. J. Control 77(1), 45-54 (2004)

32. Oppenheim, A.V., Schafer, R.W.: Discrete-Time Signal Processing, 3rd edn. Prentice Hall, Englewood Cliffs (2009)

33. Wang, D., Ye, Y., Zhang, B.: Practical Iterative Learning Control with Frequency Domain Design and Sampled Data Implementation. Springer, Singapore (2014)

34. Freeman, C.T., Lewin, P.L., Rogers, E., Owens, D.H., Hatonen, J.J.: Discrete Fourier transform based iterative learning control design for linear plants with experimental verification. J. Dyn. Syst. Meas. Control 131(3), 031006 (2009)

35. Alsubaie, M.A., Freeman, C.T., Cai, Z., Lewin, P., Rogers, E.: ILC initial input selection with experimental verification. In: Proc. IEEE Symposium on Learning Control at IEEE CDC, pp. 1-6 (2009)

36. Freeman, C.T., Alsubaie, M.A., Cai, Z., Rogers, E., Lewin, P.L.: Model and experience-based initial input construction for iterative learning control. Int. J. Adapt. Control Signal Process. 25(5), 430-447 (2011)

37. Meng, D., Jia, Y., Du, J., Yu, F.: Robust learning controller design for MIMO stochastic discrete-time systems: an $H_{\infty}$-based approach. Int. J. Adapt. Control Signal Process. 25(7), 653-670 (2011)

38. Owens, D.H., Hatonen, J.J., Daley, S.: Robust monotone gradient-based discrete-time iterative learning control. Int. J. Robust Nonlinear Control 19(6), 634-661 (2010)

39. Paszke, W., Rogers, E., Galkowski, K.: Robust finite frequency design of iterative learning control schemes. IFAC-PapersOnLine 49(13), 169-174 (2016)

40. Ge, X., Stein, J.L., Ersal, T.: A frequency-dependent filter design approach for norm-optimal iterative learning control and its fundamental trade-off between robustness, convergence speed and steady state error. J. Dyn. Syst. Meas. Control 140(2), 021004 (2018). https://doi.org/10.1115/1.4037271

41. Ardakani, M.M.G., Khong, S.Z., Bo, B.: On the convergence of iterative learning control. Automatica 78, 266-273 (2017)

\section{Submit your manuscript to a SpringerOpen ${ }^{\circ}$ journal and benefit from:}

- Convenient online submission

- Rigorous peer review

Open access: articles freely available online

- High visibility within the field

- Retaining the copyright to your article

Submit your next manuscript at $\boldsymbol{~ s p r i n g e r o p e n . c o m ~}$ 